Article

\title{
Dechdigliotoxins A-C, Three Novel Disulfide-Bridged Gliotoxin Dimers from Deep-Sea Sediment Derived Fungus Dichotomomyces cejpii
}

\author{
Zhaoming Liu, Zhen Fan, Zhanghua Sun, Hongxin Liu * and Weimin Zhang * (1) \\ State Key Laboratory of Applied Microbiology Southern China, Guangdong Provincial Key Laboratory of \\ Microbial Culture Collection and Application, Guangdong Open Laboratory of Applied Microbiology, \\ Guangdong Institute of Microbiology, Guangdong Academy of Sciences, 100 Central Xianlie Road, Yuexiu \\ District, Guangzhou 510070, China; liuzm@gdim.cn (Z.L.); zfan@sibs.ac.cn (Z.F.); sunzh@gzucm.edu.cn (Z.S.) \\ * Correspondence: liuhx@gdim.cn (H.L.);wmzhang@gdim.cn (W.Z.)
}

Received: 3 October 2019; Accepted: 21 October 2019; Published: 23 October 2019

\begin{abstract}
Dechdigliotoxins A-C (1-3), which represented the first examples of gliotoxin dimers with an unprecedented exocyclic disulfide linkage, were obtained from a deep-sea derived fungus Dichotomomyces cejpii FS110. The structures of these compounds were elucidated on the basis of spectroscopic analysis and the absolute configurations were unambiguously determined through quantum chemical calculations, as well as DP4+ probability simulations. The proposed biosynthetic pathway suggested 1-3 were generated from unusual L-Phe and D-Ser. All the isolates were evaluated for their cytotoxicity against four tumor cell lines.
\end{abstract}

Keywords: dechdigliotoxins; disulfide-bridged; dimer; deep-sea derived fungus; quantum chemical calculations; cytotoxicity

\section{Introduction}

Gliotoxin (GT), which represents the most important epidithiodioxopiperazine (ETP) type fungal toxin, is considered a lead agent for anticancer drugs due to its potent cytotoxicity $[1,2]$. Since the first description by Weindling and Emerson as a metabolite from Trichoderma lignorum [3], gliotoxin and its derivatives have been discovered from various fungal species, such as bi(dethio)-10a-methylthio-3a-deoxy-3,3a-didehydrogliotoxin and 6-deoxy-5a,6-didehydrogliotoxin from Aspergillus fumigatus [2], bisdethiobis (methylthio) gliotoxin from Gliocladium deliquescens [4], and the dimeric eutypellizines N-S from Eutypella sp [5]. Furthermore, GT derivatives are well known for their diverse bioactivities, especially the cytotoxicity. Researchers who have focused on the anticancer mechanism of GT derivatives on different cancer cell lines indicated that the intramolecular disulfide bridge makes the most influential contribution to their activities [6]. Nevertheless, to date, to the best of our knowledge, there is no relevant report about gliotoxin dimers which connect through inter-monomer disulfide bridge.

Deep-sea fungi, inhabiting the sediment or water at a depth over $1000 \mathrm{~m}$ below the surface, exhibit the potential to metabolize bioactive natural products with fantastic skeletons [7]. Among them, over $50 \%$ show cytotoxicity against different human cancer cell lines [7], for example, the cladosins I from Cladosporium sphaerospermum can suppress HL-60 cell line with the $\mathrm{IC}_{50}$ value of $2.8 \mu \mathrm{M}$ [8]. Our previous studies suggested that a strain of Dichotomomyces cejpii FS110, isolated from deep-sea sediments from South China Sea, tended to produce novel cytotoxic gliotoxin derivatives while being cultured in PDB medium [9]. In this study, further chemical and biological investigations of the extract from solid culture medium of this strain led to the discovery of three dimeric gliotoxin derivatives, 
dechdigliotoxins A-C (1-3), which represented the first examples of gliotoxin dimers constructing an unprecedented exocyclic disulfide linkage, as well as four previously obtained monomers (4-7). Herein, the details of the isolation, structure identification, and biological assay of the isolated compounds are discussed.

\section{Results and Discussion}

The solid fermentation product of the deep-sea-derived fungus Dichotomomyces cejpii FS110 was extracted with methanol and then concentrated under reduced pressure to give an extract, which was further subjected to repeated column chromatography to afford compounds 1-7 (Figure 1). The known compounds were identified as dichotocejpins $C$ and $B$ ( 4 and 5 , respectively), as well as gliotoxin and acetylgliotoxin ( 6 and 7 , respectively) by comparing their spectroscopic data with those previously isolated [9].

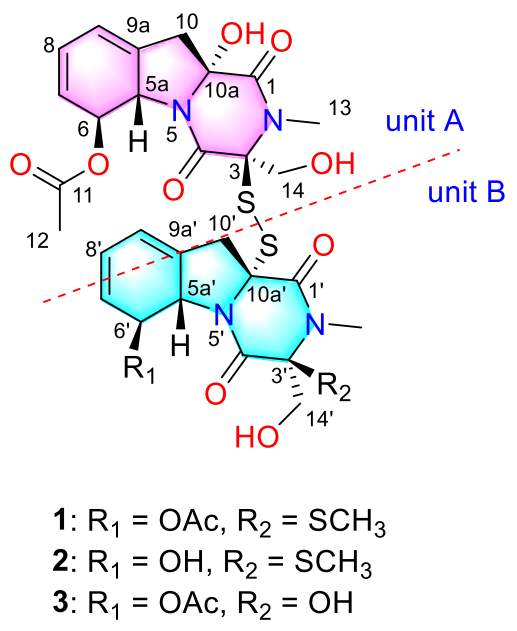

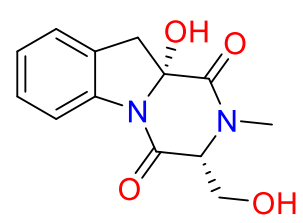

4

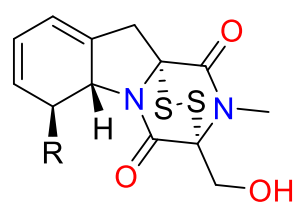

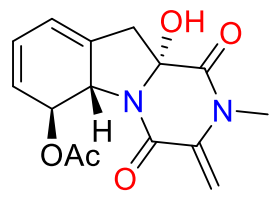

5

6: $\mathrm{R}=\mathrm{OH}$

7: $\mathrm{R}=\mathrm{OAC}$

Figure 1. The structures of 1-7.

Dechdigliotoxin A (1) was obtained as a colorless powder with molecular formula of $\mathrm{C}_{31} \mathrm{H}_{36} \mathrm{~N}_{4} \mathrm{O}_{11} \mathrm{~S}_{3}$ established on the basis of the sodiated HRESIMS peak at $\mathrm{m} / \mathrm{z} 759.1454[\mathrm{M}+\mathrm{Na}]^{+}$ (calcd 759.1440, $\mathrm{C}_{31} \mathrm{H}_{36} \mathrm{~N}_{4} \mathrm{O}_{11} \mathrm{~S}_{3} \mathrm{Na}$ ). The ${ }^{1} \mathrm{H}$ NMR spectrum recorded in DMSO (Table S1) gave the presence of a quaternary hydroxy groups at $\delta_{\mathrm{H}} 6.97(10 \mathrm{a}-\mathrm{OH}, \mathrm{s})$ and two primary alcohol hydroxy groups at $\delta_{\mathrm{H}} 5.28(14-\mathrm{OH}, \mathrm{t}, J=5.8)$ and $5.40\left(14^{\prime}-\mathrm{OH}, \mathrm{dd}, J=6.5,5.4\right)$; five methyls including two $\mathrm{N}$-methyls at $\delta_{\mathrm{H}} 3.03$ and $2.96\left(13 / 13^{\prime}-\mathrm{CH}_{3}\right.$, s); and two hydroxymethyl groups at $\delta_{\mathrm{H}} 4.33 / 3.85\left(14-\mathrm{CH}_{2}\right.$, dd) and 4.05/3.61 $\left(14^{\prime}-\mathrm{CH}_{2}, \mathrm{dd}\right) .{ }^{13} \mathrm{C} \mathrm{NMR}$, as well as HSQC spectra, revealed 31 signals, which could be divided into two sets, each containing five methines, two methyls, two methylene groups, and six quaternary carbons (including three carbonyl carbons). Comprehensive analysis of the 1D NMR and HRESIMS data suggested that dechdigliotoxin A was an unsymmetic dimeric derivative. The characteristic absorption band at $566 \mathrm{~cm}^{-1}$ in IR spectrum indicated the existence of a disulfide bond structure feature.

The complete structure was further elucidated by the COSY and HMBC correlations (Figure 2). In unit $\mathrm{A}$, the two independent coupling fragments were deduced by the ${ }^{1} \mathrm{H},{ }^{1} \mathrm{H}$-COSY correlations of $\mathrm{H}-5 \mathrm{a} / \mathrm{H}-6 / \mathrm{H}-7 / \mathrm{H}-8 / \mathrm{H}-9$ and $\mathrm{H}_{2}-14 / 14-\mathrm{OH}$. The HMBC cross-peaks from $10 \mathrm{a}-\mathrm{OH}$ to $\mathrm{C}-1, \mathrm{H}_{3}-13$ to $\mathrm{C}-1 / \mathrm{C}-3$, as well as $\mathrm{H}_{2}-14$ to $\mathrm{C}-3 / \mathrm{C}-4$, indicated the presence of diketopiperazine moiety. Furthermore, the correlations from $\mathrm{H}-5 \mathrm{a}$ to $\mathrm{C}-9 / \mathrm{C}-9 \mathrm{a}$ and $\mathrm{H}_{2}-10$ to $\mathrm{C}-5 \mathrm{a} / \mathrm{C}-9 / \mathrm{C}-9 \mathrm{a}$ in HMBC spectrum constructed a gliotoxin derived skeleton of $\mathbf{1}$. The acetyl was linked to $\mathrm{C}-6$ based on the detected correlation between $\mathrm{H}-6$ to $\mathrm{C}-11$. In unit $\mathrm{B}$, the COSY and HMBC data indicated the same skeleton as that in unit A. The main difference was that an additional methyl was connected to $C-3^{\prime}$ via a sulfur atom, which was evidenced by the chemical shifts and the only HMBC correlation from S-methyl to C-3'. Hence, the planar structure was deduced as shown. 


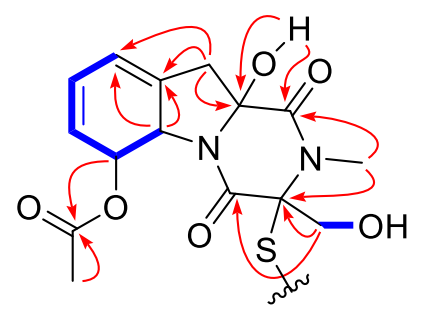

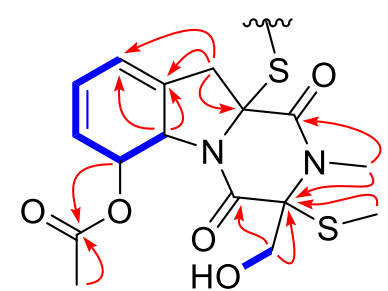
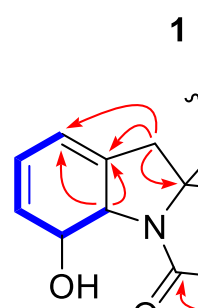

1

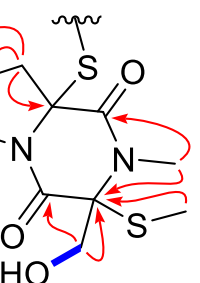

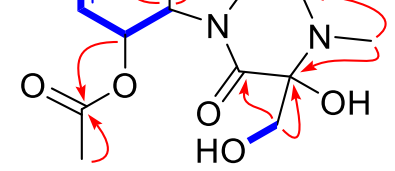

3

Unit A $:$ Unit B

2

Figure 2. Key HMBC (red arrows) and ${ }^{1} \mathrm{H},{ }^{1} \mathrm{H}-\mathrm{COSY}$ (blue bold bond) correlation of 1-3.

In order to elucidate the relative configuration of $\mathbf{1}$, the NOESY spectrum (Figure 3) was recorded in DMSO. In unit $\mathrm{A}$, the lack of correlations between $\mathrm{H}_{2}-10$ and $\mathrm{H}_{2}-14$ combined with the cross peaks of $10 \mathrm{a}-\mathrm{OH} / \mathrm{H}_{2}-14$ suggested the same side of $10 \mathrm{a}-\mathrm{OH}$ and the hydroxymethyl, which were assigned to be $\alpha$-oriatation. The NOE effect between $\mathrm{H}_{2}-10$ and $\mathrm{H}-5$ a was detected, indicating the $\beta$-oriatation of $\mathrm{H}-5 \mathrm{a}$. Finally, the large coupling constant $(\mathrm{J}=14.3 \mathrm{~Hz})$ and the correlation of $\mathrm{H}-6 / 10 \mathrm{a}-\mathrm{OH}$ confirmed the trans configuration of $\mathrm{H}-5 \mathrm{a}$ and $\mathrm{H}-6$. In unit $\mathrm{B}$, the chirality of $\mathrm{C}-5 \mathrm{a}^{\prime}, \mathrm{C}-6^{\prime}, \mathrm{C}-10 \mathrm{a}^{\prime}$ could be easily deduced through NOE correlations and coupling constant, which were the same as those in unit A. Additionally, the correlations between $\mathrm{H}_{2}-14^{\prime}$ and $\mathrm{H}-6^{\prime}$ revealed the co-facial of them.

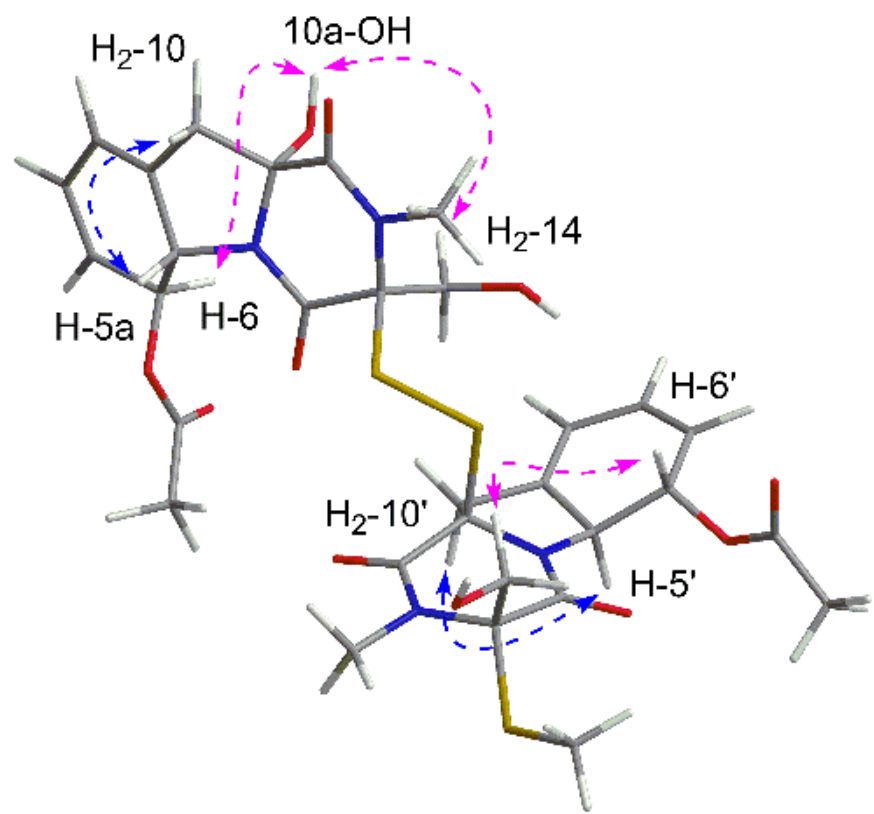

Figure 3. Key NOESY correlations of $\mathbf{1}$.

Because of the lack of convincible NOE correlations, the relative configuration between the two units could not be determined exactly. Hence, the theoretical ${ }^{13} \mathrm{C}$ NMR chemical shifts calculations and the DP4+ probability simulation of two possible relative structures (1a and $\mathbf{1 b})$ were carried 
out. Conformers within the $5 \mathrm{kcal} \mathrm{mol}^{-1}$ energy window were generated to the b3lyp/6-31+g(d,p) level. Optimized conformers with the Boltzmann distribution over $2 \%$ were chosen for NMR chemical shifts calculations in dimethyl sulphoxide at the mPW1PW91/6-311+g(d,p) level. The result (Figure 4) indicated that 1a exhibited a better correlation coefficient $\left(R^{2}: 0.9972\right)$ and mean absolute errors (MAE) value $(9.5 \mathrm{ppm})$ as compared with those of $\mathbf{1 b}\left(\mathrm{R}^{2}: 0.9948\right.$; MAE $\left.=10.0 \mathrm{ppm}\right)$. Moreover, DP4+ simulation suggested 1a should be the true structure with $99.89 \%$ probability. Then, the absolute configuration of $\mathbf{1}$ was elucidated by performing a quantum chemical calculation of the ECD spectra at the b3lyp/6-311+g(d,p) level. The optimized conformers of both $\mathbf{1 a}$ and $\mathbf{1 b}$ were selected based on the previous NMR calculations. The results shown in Figure 5 suggested that the calculated plot of $\mathbf{1 a}$ exhibited a better fit to the experimental spectrum. Thus, the absolute configuration was assigned as $3 S, 5 a S, 6 S, 10 a R, 3^{\prime} S, 5 a^{\prime} S, 6^{\prime} S$, and $10 a^{\prime} R$.
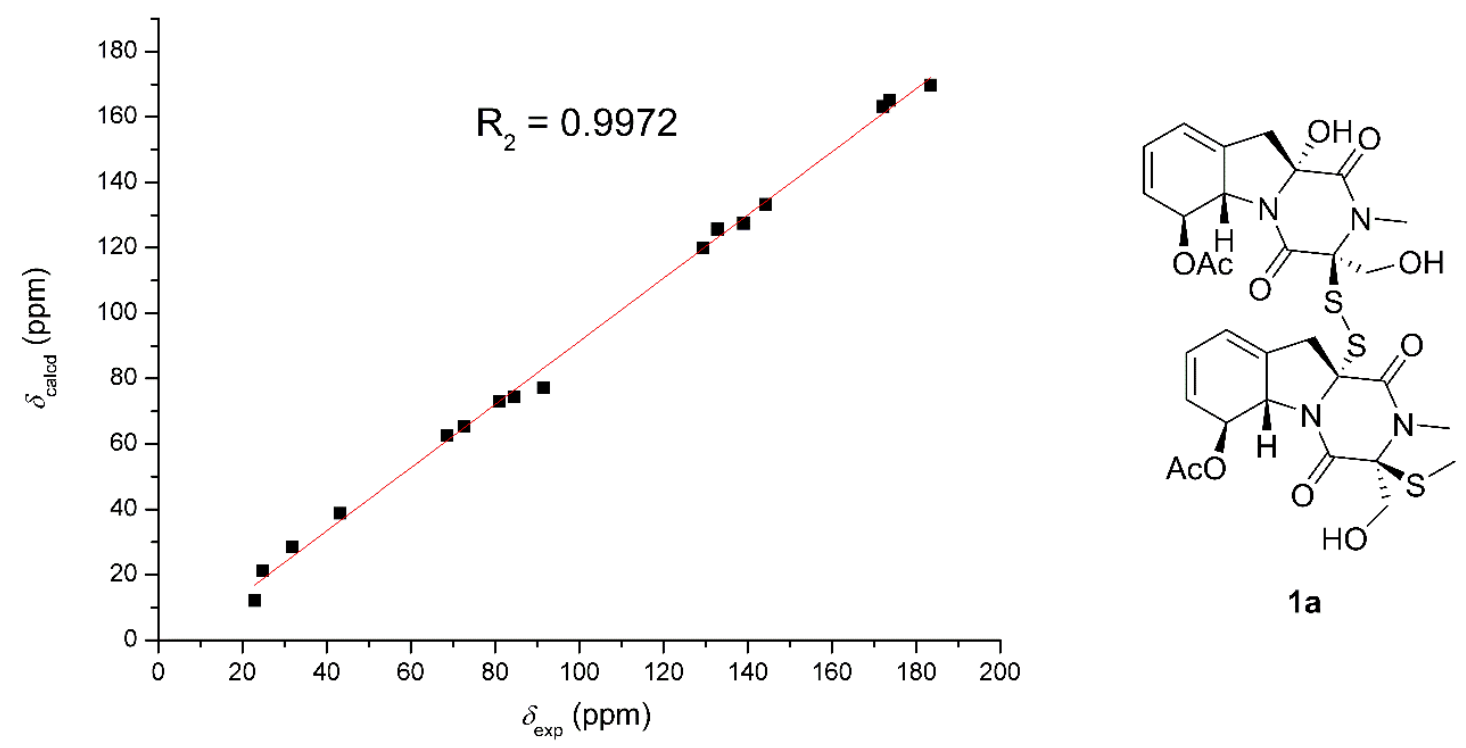

1a
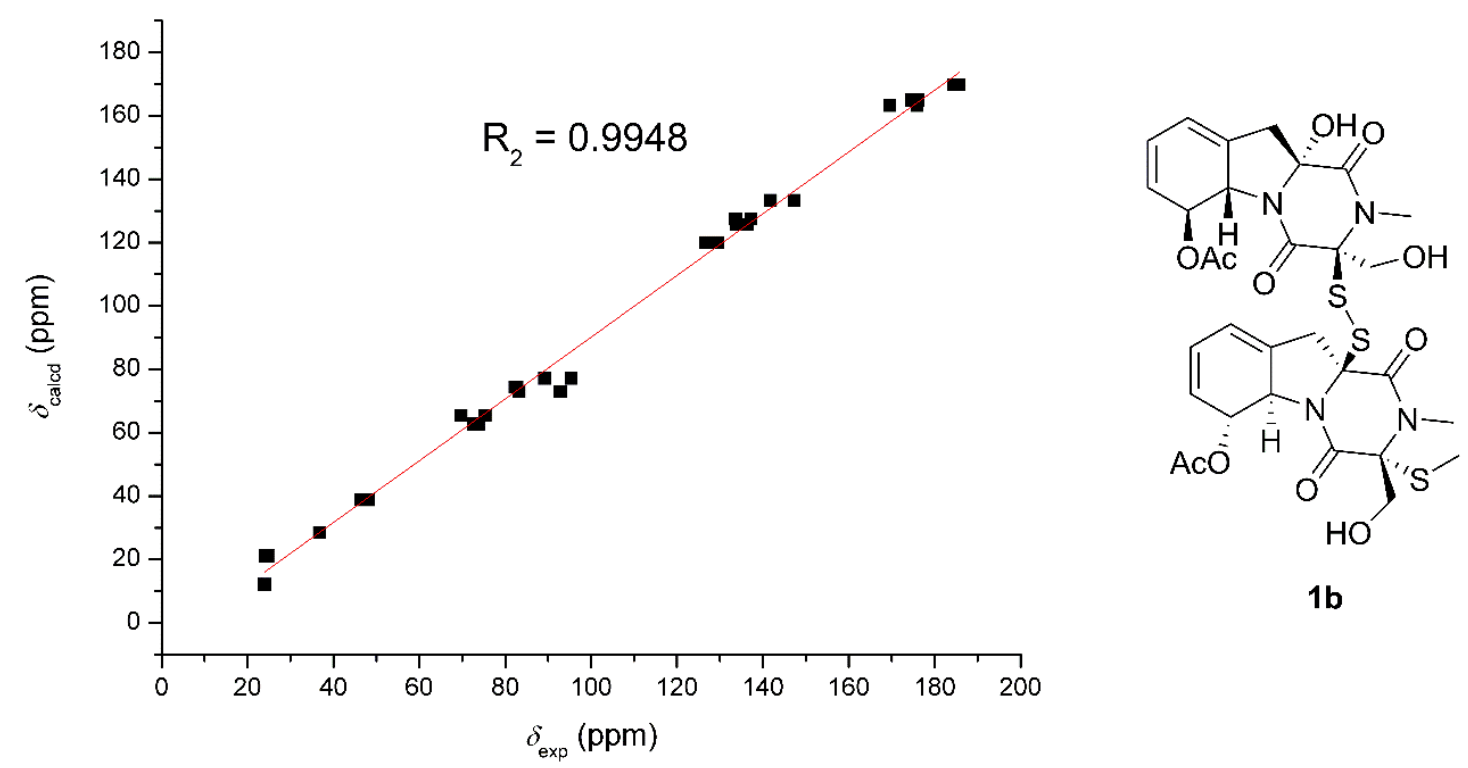

1b

Figure 4. Correlation plots of experimental and calculated ${ }^{13} \mathrm{C} N M R$, as well as DP4+ simulation of 1a and $\mathbf{1 b}$. 


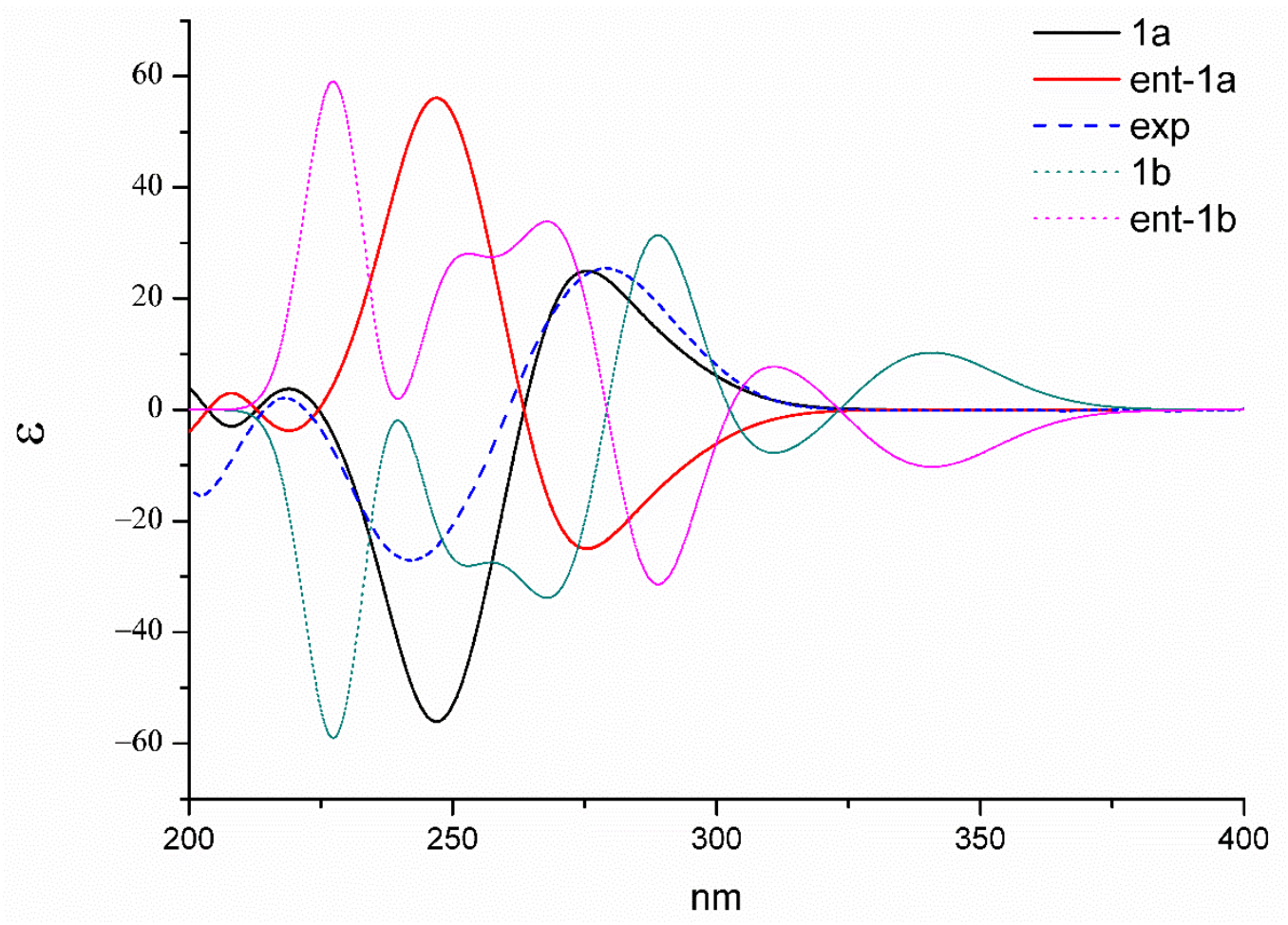

Figure 5. Experimental and calculated ECD spectra of $\mathbf{1 a}$ /ent-1a and $\mathbf{1 b} / \mathbf{e n t}-\mathbf{1 b}$.

Dechdigliotoxin B (2), which was obtained as a colorless powder, gave the molecular formula of $\mathrm{C}_{29} \mathrm{H}_{34} \mathrm{~N}_{4} \mathrm{O}_{10} \mathrm{~S}_{3}$ based on the sodiated HRESIMS peak at m/z 717.1323 [M $\left.+\mathrm{Na}\right]^{+}$(calcd 717.1335, $\mathrm{C}_{29} \mathrm{H}_{34} \mathrm{~N}_{4} \mathrm{O}_{10} \mathrm{~S}_{3} \mathrm{Na}$ ). The ${ }^{1} \mathrm{H}$ and ${ }^{13} \mathrm{C}$ NMR spectra recorded in methanol were nearly identical to those of 1 and the main difference was that the methyl signal $\delta_{\mathrm{H}} 2.00\left(12^{\prime}-\mathrm{Me}\right)$ and the carbonyl carbon $\delta_{\mathrm{C}} 169.8\left(\mathrm{C}-11^{\prime}\right)$ were absent. Moreover, the obvious upfield shifts of $\mathrm{H}-5 \mathrm{a}^{\prime}$ and $\mathrm{H}-6^{\prime}$ (from $\delta_{\mathrm{H}-5 \mathrm{a}^{\prime}}$ 5.18 and $\delta_{\mathrm{H}-6^{\prime}} 6.14$ in 1 to $\delta_{\mathrm{H}-5 \mathrm{a}^{\prime}} 4.96$ and $\delta_{\mathrm{H}-6^{\prime}} 4.86$ in 2, respectively) were observed, suggesting that dechdigliotoxin B was a deacetylation product of $\mathbf{1}$ at C- $6^{\prime}$. Further analysis of 2D NMR data confirmed the planar structure as shown (Figure 2).

The relative configuration was elucidated based on the NOESY correlations recorded in dimethyl sulfoxide- $d_{6}$ (Figure 6) and the coupling constants, which were the same as those of dechdigliotoxin A. Additionally, the identical cotton effects in the experimental ECD spectra (Figure 7) shared by $\mathbf{1}$ and $\mathbf{2}$ indicated the same absolute configuration of them. Hence, the complete structure of dechdigliotoxin B was deduced.
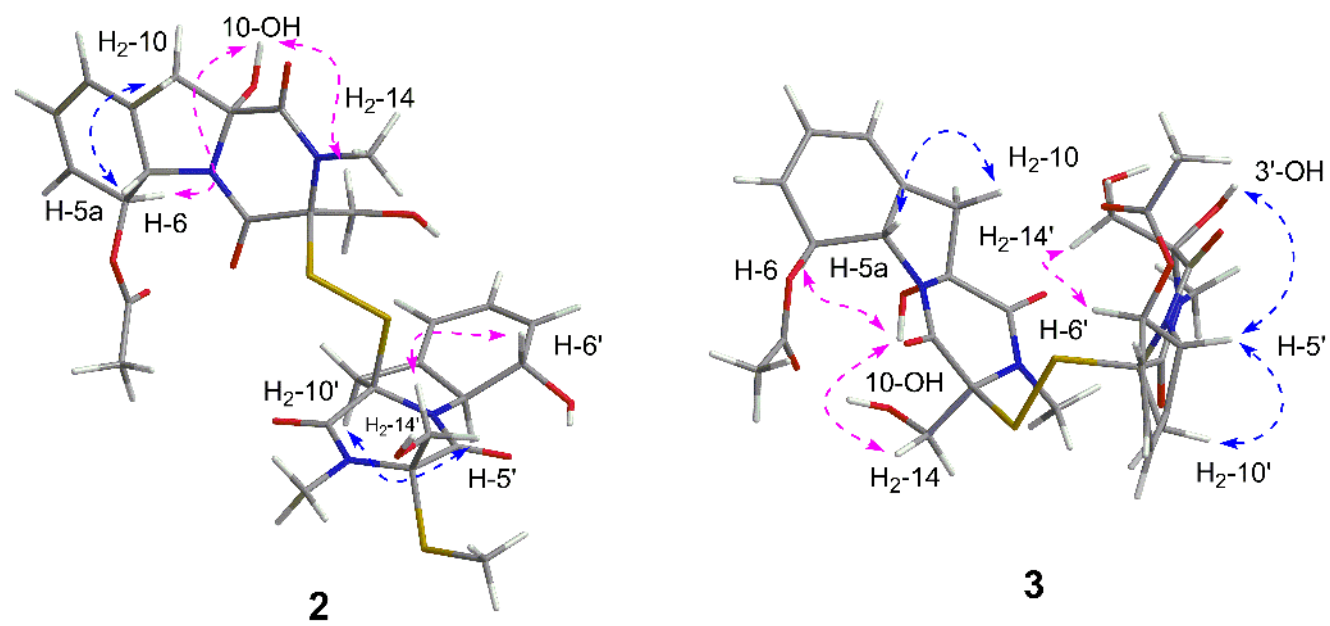

3

Figure 6. Key NOESY correlations of $\mathbf{2}$ and $\mathbf{3}$ recorded in DMSO. 


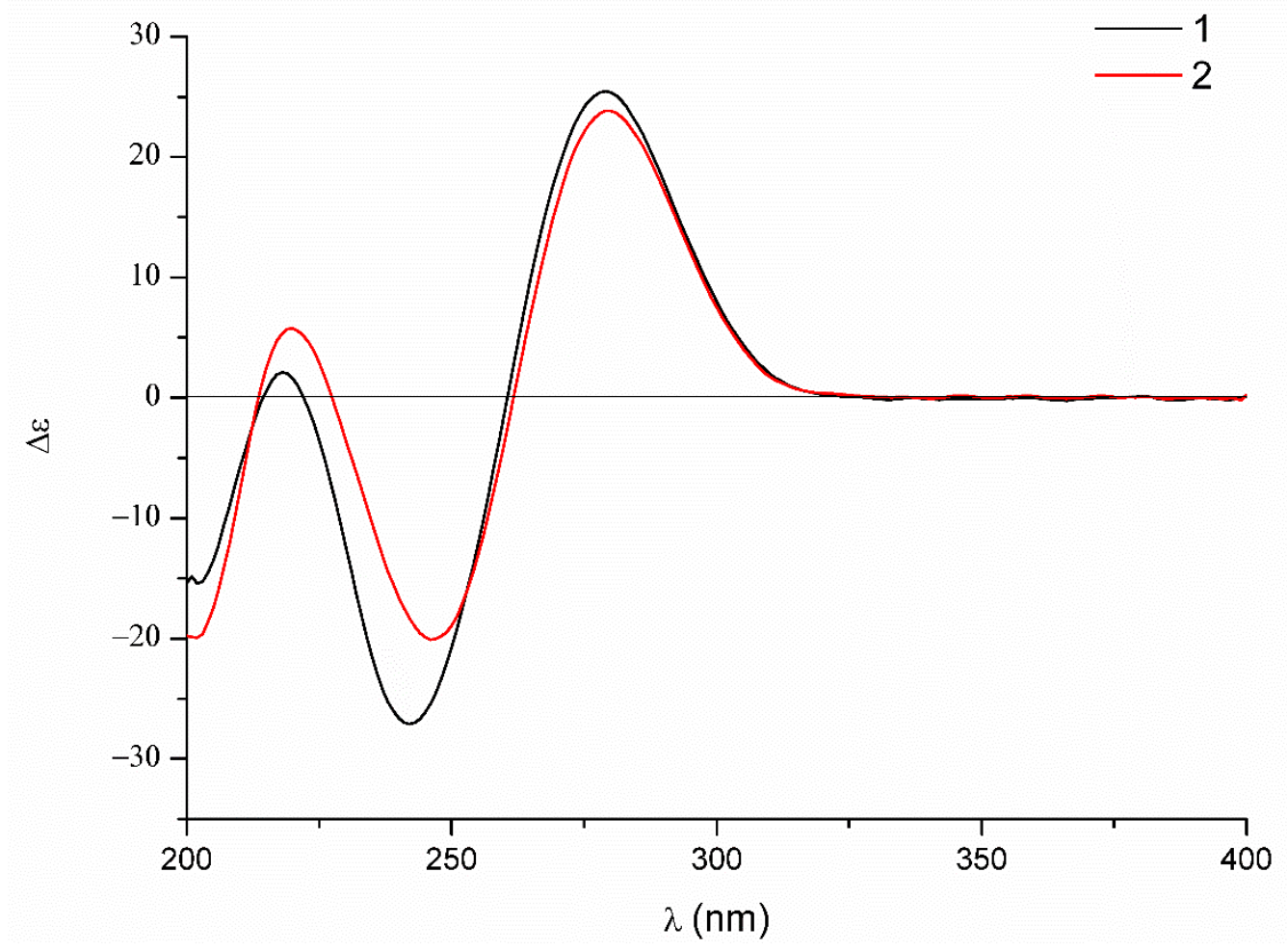

Figure 7. Experimental ECD spectra of $\mathbf{1}$ and 2.

Dechdigliotoxin C (3) was isolated as a colorless powder, of which the molecular formula was deduced as $\mathrm{C}_{30} \mathrm{H}_{34} \mathrm{~N}_{4} \mathrm{O}_{12} \mathrm{~S}_{2}$ on the basis of the sodiated ion peak at $\mathrm{m} / \mathrm{z} 729.1519[\mathrm{M}+\mathrm{Na}]^{+}$(calcd 717.1615, $\mathrm{C}_{30} \mathrm{H}_{34} \mathrm{~N}_{4} \mathrm{O}_{12} \mathrm{~S}_{2} \mathrm{Na}$ ) in HRESIMS. The similar 1D NMR data (Table 1) and the characteristic IR absorption band at $555 \mathrm{~cm}^{-1}$ as compared to those of 1 suggested that they shared the same framework except the substituent at $C-3^{\prime}$. The absence of S-methyl $\left(\delta_{\mathrm{H}} 2.11 ; \delta_{\mathrm{C}} 13.0\right)$ and the downfield shift of $\mathrm{C}-3^{\prime}\left(\delta_{\mathrm{C}} 88.2\right)$ indicated that the SMe in unit $\mathrm{B}$ was replaced by a hydroxy group. The further HMBC correlations (Figure 2) from $\mathrm{H}_{3}-13^{\prime}$ to $\mathrm{C}-1^{\prime} / \mathrm{C}-3^{\prime}$ and from $\mathrm{H}_{2}-14^{\prime}$ to $\mathrm{C}-3^{\prime} / \mathrm{C}-4^{\prime}$ confirmed the planar structure of dechdigliotoxin $\mathrm{C}$. The relative configurations of unit A and B were elucidated to be the same as those of $\mathbf{1}$ and $\mathbf{2}$ on the basis of NOESY correlation measured in dimethyl sulfoxide- $d_{6}$ (Figure 6).

Table 1. In vitro cytotoxicity against tested cancer cell lines of 1-7.

\begin{tabular}{ccccc}
\hline \multirow{2}{*}{ Compounds } & \multicolumn{4}{c}{ IC $_{50}(\mu \mathbf{M})$} \\
\cline { 2 - 5 } & SF-268 & MCF-7 & NCI-H460 & HepG-2 \\
\hline $1-5$ & $>100$ & $>100$ & $>100$ & $>100$ \\
6 & $0.24 \pm 0.10$ & $0.08 \pm 0.00$ & $0.24 \pm 0.01$ & $0.21 \pm 0.01$ \\
7 & $0.25 \pm 0.03$ & $0.22 \pm 0.04$ & $0.32 \pm 0.02$ & $0.49 \pm 0.07$ \\
Cisplatin & $2.37 \pm 0.35$ & $3.09 \pm 0.27$ & $2.43 \pm 0.15$ & $1.39 \pm 0.18$ \\
\hline
\end{tabular}

The relative structure between the two monomers were determined based on the ${ }^{13} \mathrm{C}$ NMR chemical shift calculations and DP4+ simulation (Figure 8). The method used was the same as those adopted in $\mathbf{1}$. The $\mathrm{R}^{2}$ of $\mathbf{3 a}$ was 0.9935 , which was slightly better than that of $\mathbf{3 b}$ (0.9926). DP4+ (chemical shift of carbon data) suggested 3a should be the likely candidate with $99.5 \%$ probability. Finally, the identical experimental ECD spectra suggested the same absolute configuration of 3 . In order to further confirm the stereochemistry of dechdigliotoxin $C$, the theoretical ECD spectra of the two enantiomers (3a and ent-3a) were also calculated at the b3lyp/6-311+g(d,p) level (Figure 9). 
The experimental spectrum exhibited an excellent fit to the theoretical plot of 3 , which confirmed the absolute configuration.
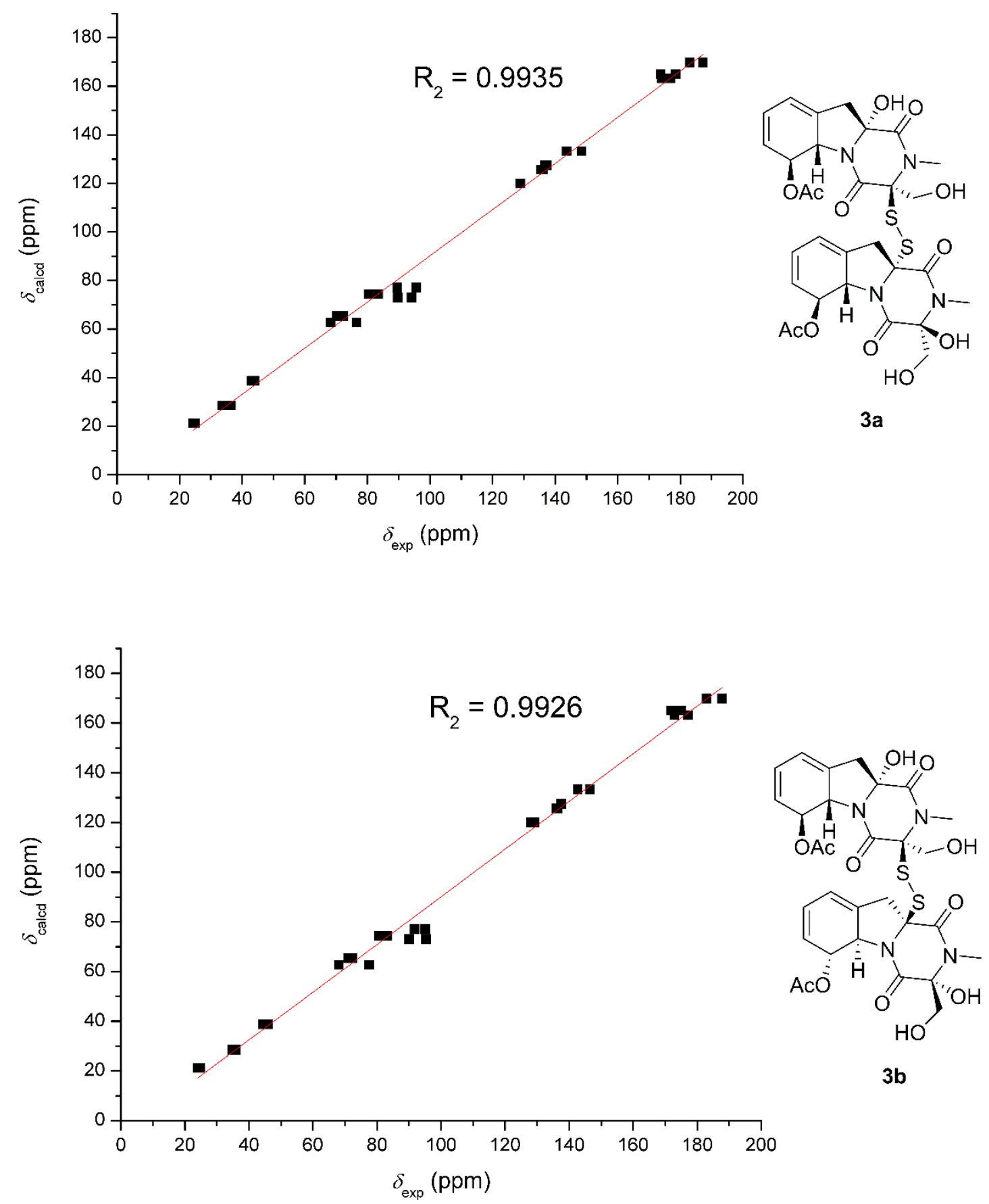

Figure 8. Correlation plots of experimental and calculated ${ }^{13} \mathrm{C}$ NMR, as well as DP4+ simulation of 3a and $3 \mathbf{b}$. 


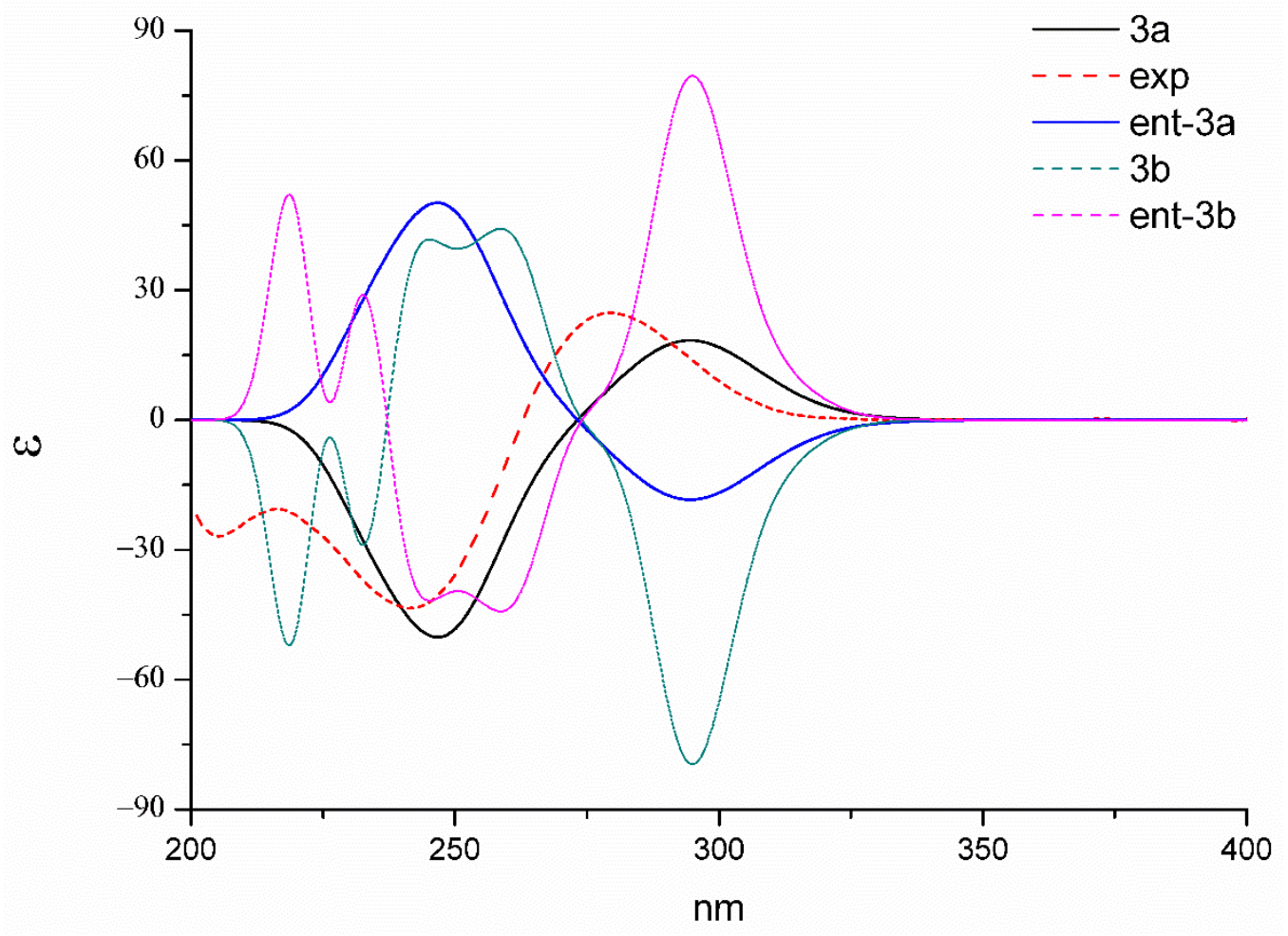

Figure 9. Experimental and calculated ECD spectra of $3 \mathbf{a} /$ ent-3a and $3 \mathbf{b} / \mathrm{ent}-\mathbf{3 b}$.

Cyclic phenylalanylserine cyclo (L-Pshe-L-Ser) is a known biosynthetic precursor of the epidithiodioxopiperazine gliotoxin [10], however, the obtained new metabolites, dechdigliotoxins A-C, in this study, which exhibited a converse chirality at C- $3^{\prime}$ as compared with those reported gliotoxin analogues, were suspected to be generated from unusual L-Phe and D-Ser. The possible biosynthesis pathway was shown in Scheme 1. The cyclo-phenylalanyl-serine (L-Phe-D-Ser) was constructed through the dipeptidase. Then, the hydroxylation of $\alpha$-protons of the two amino acid were performed with retention of the chirality of C-3' and C-10a' (intermediate i), which would metabolize 4 and 5 . The glutathione-S-transferase should be the key enzyme contributed to the sulfuriztion at C- $3^{\prime}$ or/and $\mathrm{C}-10 \mathrm{a}^{\prime}$, giving intermediates ii and iii. Followed by the monooxygenase and O-methyltransferase, the two precursor units A and B were formed from intermediates $\mathbf{i}$ and ii. Finally, the formation of disulfide bond between the two units which constructed 1-3 was catalyzed by oxidoreductase. Since the isolated dimers (1-3) and the monomer (5, of which the absolute configuration was confirmed by X-ray diffraction, no. CCDC 1491672, Figure S28) shared the same precursor of intermediate i, the stereochemistry could be further evidenced by the same biogenetic consistency.

As it is known that gliotoxin exhibits strong cytotoxicity against cancer cell lines, as well as the normal cell lines, hence, the dimeric analogues 1-7 were evaluated for their cytotoxic effects against SF-268, MCF-7, HepG-2, and NCI-H460 cancer cell lines using cisplatin as a positive control. However, none of them showed effects against the tested cell lines except for the gliotoxin (6) and acetyl-gliotoxin (7) (Table 1). According to the primary structure-activity relationship, we speculated that when the intramolecular disulfide bridge was deficient or converted to an "intermolecular-like" in the dimer, the cytotoxicity would reduce significantly. This would offer a new idea to reduce the toxicity of gliotoxin derivatives toward the normal cell lines. On the other hand, more bioactivities, not limited to the cytotoxicity, would be screened for the new dimers 1-3 in the future. 


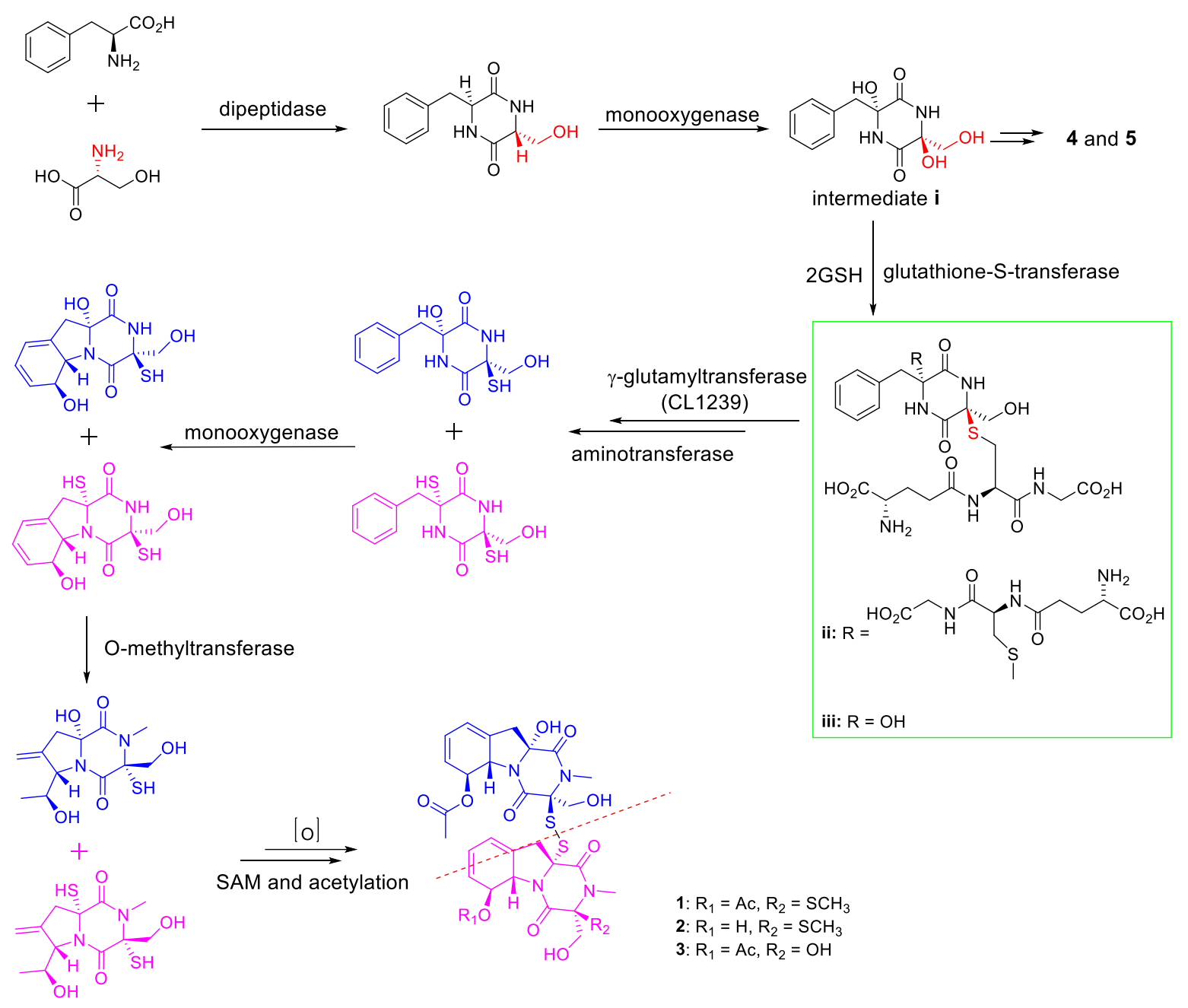

Scheme 1. Possible biosynthesis pathway of 1-3.

\section{Materials and Methods}

\subsection{General}

HRESIMS were measured on a Thermo MAT95XP high resolution mass spectrometer (Thermo Fisher Scientific, Bremen, Germany). Optical rotations were recorded using an Anton Paar MCP-500 (Anton Paar, Graz, Austria) and the electronic circular dichroism (ECD) measured on a Jasco 820 spectropolarimeter (Jasco Corporation, Kyoto, Japan) under $\mathrm{N}_{2}$ gas protection. IR and UV spectra were recorded on Shimadzu IR Affinity-1 and Shimadzu UV-2600 (Shimadzu Corporation, Kyoto, Japan) spectrophotometer, respectively. NMR measurements were carried out on a Bruker Avance-500M spectrometer (Bruker, Fällanden, Switzerland) with tetramethylsilane as an internal standard. Shimadzu LC-20 AT (Shimadzu Corporation, Kyoto, Japan) equipped with an SPD-M20A PDA detector (Shimadzu Corporation, Kyoto, Japan) was adopted for semipreparative HPLC separation with YMC-pack SIL and YMC-pack ODS-A column $(250 \times 20$ mm, 5 mm, 12 nm, YMC CO., Ltd., Kyoto, Japan). Silica gel (200-300 mesh) and Sephadex LH-20 gel were purchased from Qingdao Marine Chemical Plant (Qingdao, China) and Amersham Biosciences (Uppsala, Sweden), respectively. All solvents were of analytical grade (Guangzhou Chemical Regents Company, Ltd., Guangzhou, China).

\subsection{Material and Identification}

The fungal strain D. cejpii FS110 was isolated from deep-sea sludge in the South China Sea (19 $0.368^{\prime} \mathrm{N}, 117^{\circ} 58.233^{\prime} \mathrm{E}$; depth $3941 \mathrm{~m}$ ), which was identified as Dichotomomyces cejpii according to 
morphological traits and ITS rDNA sequence analysis. The sequence data have been submitted to GenBank, accession number KF706672. The details of the genetic and deposit information could be found in our previously published reference [9].

\subsection{Fermentation, Extraction, and Isolation}

A grown PDA culture of D. cejpii FS110 was used for preparation of the seed cultures, which was further inoculated in $250 \mathrm{~mL}$ of $\mathrm{PDB}$ (potato $200 \mathrm{~g} / \mathrm{L}$, glucose $20 \mathrm{~g} / \mathrm{L}, \mathrm{KH}_{2} \mathrm{PO}_{4} 3 \mathrm{~g} / \mathrm{L}, \mathrm{MgSO}_{4} \bullet 7 \mathrm{H}_{2} \mathrm{O}$ $1.5 \mathrm{~g} / \mathrm{L}$, vitamin $B_{1} 10 \mathrm{mg} / \mathrm{L}$, sea salt $15 \mathrm{~g} / \mathrm{L}$ ) in a $250 \mathrm{~mL}$ Erlenmeyer flask. After incubating for 5 days in a rotary shaker $(200 \mathrm{rpm})$ at $28^{\circ} \mathrm{C}$, the seed culture was transformed to solid medium (30 Erlenmeyer flask each containing $250 \mathrm{~g}$ rice and $400 \mathrm{~mL}$ saline water). After stationary incubating for 30 days at room temperature, the fermentation product was extracted with methanol to yield a dark brown oily residue ( $40.0 \mathrm{~g}$ ), which was further subjected to column chromatography on silica gel eluted with petroleum ether/EtOAc in linear gradient (9:1 to 1:3) to give 26 fractions (Fr.1-Fr.26). Fr.7 was subjected to Sephdex-20 washed by methanol to obtain compound 1 (4.8 mg). Fr.9 was subjected to Sephadex LH-20 (methanol/dichloromethane, 1/1, v/v) to give compound 2, which was further purified by semi-preparative reversed-phase (RP) HPLC system equipped with a YMC column (methanol/water, 85:15, $2 \mathrm{~mL} / \mathrm{min})\left(1.4 \mathrm{mg}, \mathrm{t}_{R}=17 \mathrm{~min}\right)$. Fr. 10 was separated by silica gel (methanol/ dichloromethane, 2/98, v/v) to yield $5(1.0 \mathrm{mg})$. Fr.11 was subjected to preparative reversed-phase (RP) HPLC system equipped with a YMC column (methanol/water, 60:40, $8 \mathrm{~mL} / \mathrm{min}$ ), then further purified by semi-preparative reversed-phase (RP) HPLC with the same column (acetonitrile/water, $90: 10,2 \mathrm{~mL} / \mathrm{min})$ to get $3\left(2.1 \mathrm{mg}, \mathrm{t}_{\mathrm{R}}=9.7 \mathrm{~min}\right)$ and $4\left(2.4 \mathrm{mg}, \mathrm{t}_{\mathrm{R}}=10.9 \mathrm{~min}\right)$.

\subsubsection{Dechdigliotoxin A (1)}

Colorless amorphous powder; $[\alpha]_{\mathrm{D}}^{25}+99.9$ (c 0.01, mathanol); UV (MeOH) $\lambda_{\max }(\log \varepsilon): 220$ (4.15), 265 (2.11) nm. IR (KBr): 3270, 2944, 2850, 1732, 1721, 1690, 1680, 1675, 1298, 1206, 1011, $566 \mathrm{~cm}^{-1}$; HRESIMS m/z 759.1454 [M + Na] ${ }^{+}\left(\right.$calcd 759.1440, $\left.\mathrm{C}_{31} \mathrm{H}_{36} \mathrm{~N}_{4} \mathrm{O}_{11} \mathrm{~S}_{3} \mathrm{Na}\right) ;{ }^{1} \mathrm{H}$ and ${ }^{13} \mathrm{C}$ NMR data: see Table S1 and Figure S1-S6.

\subsubsection{Dechdigliotoxin B (2)}

Colorless amorphous powder; $[\alpha]_{\mathrm{D}}^{25}+85.4$ (c 0.01, mathanol); UV (MeOH) $\lambda_{\max }(\log \varepsilon): 219(4.18)$, 263 (2.10) nm; IR (KBr): 3266, 2951, 2790, 1720, 1698, 1677, 1676, 1241, 1216, 1021, $563 \mathrm{~cm}^{-1}$; HRESIMS $\mathrm{m} / \mathrm{z} 717.1323[\mathrm{M}+\mathrm{Na}]^{+}\left(\right.$calcd $\left.717.1335, \mathrm{C}_{29} \mathrm{H}_{34} \mathrm{~N}_{4} \mathrm{O}_{10} \mathrm{~S}_{3} \mathrm{Na}\right) ;{ }^{1} \mathrm{H}$ and ${ }^{13} \mathrm{C}$ NMR data: see Table $\mathrm{S} 1$ and Figure S7-S13.

\subsubsection{Dechdigliotoxin C (3)}

Colorless amorphous powder; $[\alpha]_{\mathrm{D}}^{25}-109.7$ (c 0.01, methanol); UV (MeOH) $\lambda_{\max }(\log \varepsilon): 221$ (3.98),269 (1.97) nm. IR (KBr): 3199, 2898, 2760, 1720, 1709, 1688, 1682, 1679, 1240, 1206, 1031, $555 \mathrm{~cm}^{-1}$; HRESIMS m/z 729.1529 [M + Na ${ }^{+}\left(\right.$calcd 717.1615, $\left.\mathrm{C}_{30} \mathrm{H}_{34} \mathrm{~N}_{4} \mathrm{O}_{12} \mathrm{~S}_{2} \mathrm{Na}\right) ;{ }^{1} \mathrm{H}$ and ${ }^{13} \mathrm{C}$ NMR data: see Table S1 and Figure S14-S24.

\subsection{Quantum Chemical Calculation of NMR Chemical Shifts and ECD Spectrra}

Merck molecular force field (MMFF) and DFT/TD-DFT calculations were carried out using the Spartan'14 software (Wavefunction Inc., Irvine, CA, USA) and the Gaussian 09 program, respectively [11]. Conformers which had an energy window lower the $5 \mathrm{kcal} \mathrm{mol}^{-1}$ were generated and optimized using DFT calculations at the b3lyp/6-31+g(d,p) level. Frequency calculations were performed at the same level to confirm that each optimized conformer was a true minimum and to estimate their relative thermal free energy $(\Delta \mathrm{G})$ at $298.15 \mathrm{~K}$. Conformers with the Boltzmann distribution over $2 \%$ were chosen for ${ }^{13} \mathrm{C}$ NMR and ECD calculations at the mPW1PW91/6-311+g(d,p) and the b3lyp/6-311+g(d,p) level, respectively. Additionally, solvent effects were considered based on the 
self-consistent reaction field (SCRF) method with the polarizable continuum model (PCM) [12]. Details of the conformers' information were provided in Supplementary Materials (Tables S2-S5). The ECD spectrum was generated by the SpecDis program [13] using a Gaussian band shape with $0.22 \mathrm{eV}$ exponential half-width from dipole-length dipolar and rotational strengths. The DP4+ probability simulations were conducted using an applet available at http://www-jmg.ch.cam.ac.uk/tools/nmr/DP4/.

\subsection{Cytotoxicity Assay}

The tested cell lines were purchased from the Chinese Academy of Sciences Cell Bank (Shanghai, China). SF-268, MCF-7, and HepG-2 were selected to be the targeted cancer cell lines and the cytotoxicity were tested based on the previously reported SRB method [14]. Cells $(180 \mu \mathrm{L})$ with a density of 30,000 cells $/ \mathrm{mL}$ of media were injected into $96-$-well plates and incubated at $37^{\circ} \mathrm{C}$ for $24 \mathrm{~h}$ under $5 \% \mathrm{CO}_{2}$. Then, different concentrations of the tested compounds $(20 \mu \mathrm{L})$ were added and further incubated for $72 \mathrm{~h}$. After that, cell monolayers were fixed with $50 \mu \mathrm{L}$ trichloroacetic acid (wt/v: $50 \%)$ and stained with $0.4 \% \mathrm{SRB}$ (dissolved in $1 \% \mathrm{CH}_{3} \mathrm{COOH}$ ) for $30 \mathrm{~min}$. The monolayers were washed by $1 \% \mathrm{CH}_{3} \mathrm{COOH}$ three times to remove the unbound dye. The mixtures were dissolved in $200 \mu \mathrm{L}$ Tris base solution $(10 \mathrm{mM})$ and recorded the OD at $570 \mathrm{~nm}$ using a microplate reader. Cisplatin was used as a positive control possessing potent cytotoxic activity. All data were obtained in triplicate.

\section{Conclusions}

In summary, seven gliotoxin derivatives were obtained from the deep-sea derived fungus Dichotomomyces cejpii. To the best of our knowledge, the three novel dimers dechdigliotoxins A-C (1-3) represented the first examples of dimeric analogues from nature with an unprecedented exocyclic disulfide linkage. The proposed biosynthetic pathway suggested that they should be generated from the unusual precursors L-Phe and D-Ser. Moreover, the bioassays indicated that when the intramolecular disulfide bridge was deficient or converted to a "intermolecular-like" in the dimer, the cytotoxicity would reduce significantly. This discovery makes a contribution to the chemical diversity of gliotoxins and, for further investigation, offers a new idea to reduce the toxicity of gliotoxins.

Supplementary Materials: The following are available online at http:/www.mdpi.com/1660-3397/17/11/596/s1, Figure S1: ${ }^{1} \mathrm{H}$ NMR spectrum of $\mathbf{1}$ in DMSO (600 MHz), Figure S2: ${ }^{13} \mathrm{C}$ NMR spectrum of $\mathbf{1}$ in DMSO (150 MHz), Figure S3: ${ }^{1} \mathrm{H}-{ }^{1} \mathrm{H}$ COSY spectrum of $\mathbf{1}$ in DMSO $(600 \mathrm{MHz})$, Figure S4: HSQC spectrum of $\mathbf{1}$ in DMSO $(600 \mathrm{MHz})$, Figure S5: HMBC spectrum of $\mathbf{1}$ in DMSO(600 MHz), Figure S6: NOESY spectrum of $\mathbf{1}$ in DMSO (600 MHz), Figure S7: ${ }^{1} \mathrm{H}$ NMR spectrum of 2 in MeOD (600 MHz), Figure S8: ${ }^{13} \mathrm{C}$ NMR spectrum of 2 in MeOD $(600 \mathrm{MHz})$, Figure S9: ${ }^{1} \mathrm{H}-{ }^{1} \mathrm{H}$ COSY spectrum of 2 in MeOD (600 MHz), Figure S10: HSQC spectrum of 2 in MeOD $(600 \mathrm{MHz})$, Figure S11: HMBC spectrum of 2 in MeOD (600 MHz), Figure S12: ${ }^{1} \mathrm{H}$ NMR spectrum of 2 in DMSO $(600 \mathrm{MHz})$, Figure S13: NOESY spectrum of 2 in DMSO (600 MHz), Figure S14: ${ }^{1} \mathrm{H}$ NMR spectrum of 3 in MeOD (500 MHz), Figure S15: ${ }^{13} \mathrm{C}$ NMR spectrum of 3 in MeOD (500MHz), Figure S16: ${ }^{1} \mathrm{H}-{ }^{1} \mathrm{H}$ COSY spectrum of 3 in MeOD (500 MHz), Figure S17: HSQC spectrum of 3 in MeOD (500 MHz), Figure S18: HMBC spectrum of 3 in MeOD (500 $\mathrm{MHz})$, Figure S19: ${ }^{1} \mathrm{H}$ NMR spectrum of 3 in DMSO (600 MHz), Figure S20: ${ }^{13} \mathrm{CNMR}$ spectrum of 3 in DMSO $(150 \mathrm{MHz})$, Figure S21: ${ }^{1} \mathrm{H},{ }^{1} \mathrm{H}-\mathrm{COSY}$ spectrum of 3 in DMSO $(600 \mathrm{MHz})$, Figure S22: HSQC spectrum of 3 in DMSO (600 MHz), Figure S23: HMBC spectrum of 3 in DMSO (600 MHz), Figure S24: NOESY spectrum of 3 in DMSO (500 MHz), Figure S25: HRESI TOF MS spectrum of 1, Figure S26: HRESI TOF MS spectrum of 2, Figure S27: HRESI TOF MS spectrum of 3, Figure S28: The crystal structure of 4, Table S1: 1H and 13C NMR data of 1-3, Table S2: Energy analysis for the Conformers of 1a, Table S3: Energy analysis for the Conformers of $\mathbf{1 b}$, Table S4: Energy analysis for the Conformers of 3a, Table S5: Energy analysis for the Conformers of $\mathbf{3 b}$.

Author Contributions: Z.L. performed the manuscript, the quantum chemical calculations, and the structure identification; Z.F. and Z.S. isolated the metabolites from Dichotomomyces cejpii; H.L. and W.Z. designed and supervised the research and revised the manuscript.

Funding: This research was funded by National Natural Science Foundation of China $(41906106,31272087)$, the Science and Technology Program of Guangzhou, China (201607020018), the Team Project of the Natural Science Foundation of Guangdong Province (2016A030312014), and the GDAS' Project of Science and Technology Development (2019GDASYL-0103007).

Conflicts of Interest: The authors declare no conflict of interest. 


\section{References}

1. Vigushin, D.M.; Mirsaidi, N.; Brooke, G.; Sun, C.; Pace, P.; Inman, L.; Moody, C.J.; Coombes, R.C. Gliotoxin is a dual inhibitor of farnesyltransferase and geranylgeranyltransferase I with antitumor activity against breast cancer in vivo. Med. Oncol. 2004, 21, 21-30. [CrossRef]

2. Sun, Y.; Takada, K.; Takemoto, Y.; Yoshida, M.; Nogi, Y.; Okada, S.; Matsunaga, S. Gliotoxin analogues from a marine-derived fungus, Penicillium sp., and their cytotoxic and histone methyltransferase inhibitory activities. J. Nat. Prod. 2012, 75, 111-114. [CrossRef] [PubMed]

3. Wdindling, R.; Emerson, O.H. The isolation of a toxic substance from the culture filtrates of Trichoderma. Phytopath 1936, 26, 1068-1074.

4. Kirby, G.W.; Robins, D.J.; Sefton, M.A.; Talekar, R.R. Biosynthesis of bisdethiobis (methylthio) gliotoxin, a new metabolite of Gliocladium deliquescens. J. Chem. Soc. Perkin Trans. 1980, 1, 119-121. [CrossRef]

5. Niu, S.; Liu, D.; Shao, Z.; Proksch, P.; Lin, W. Eutypellazines N-S, new thiodiketopiperazines from a deep sea sediment derived fungus Eutypella sp. with anti-VRE activities. Terahedron Lett. 2017, 58, 3695-3699. [CrossRef]

6. Waring, P.; Sjaarda, A.; Linm, Q.H. Gliotoxin inactivates alcohol dehydrogenase by either covalent modification or free radical damage mediated by redox cycling. Biochem. Pharmacol. 1995, 49, 1195-1201. [CrossRef]

7. Wang, Y.; Xue, Y.; Liu, C. A brief review of bioactive metabolites derived from deep-sea fungi. Mar. Drugs. 2015, 13, 4594-4616. [CrossRef] [PubMed]

8. Wu, G.; Sun, X.; Yu, G.; Wang, W.; Zhu, T.; Gu, Q.; Li, D. Cladosins A-E, hybrid polyketides from a deep-sea-derived fungus, Cladosporium sphaerospermum. J. Nat. Prod. 2014, 77, 270-275. [CrossRef] [PubMed]

9. Fan, Z.; Sun, Z.; Liu, Z.; Chen, Y.; Liu, H.; Li, H.; Zhang, W. Dichotocejpins A-C: New Diketopiperazines from a Deep-Sea-Derived Fungus Dichotomomyces cejpii FS110. Mar. Drugs. 2016, 14, 164. [CrossRef] [PubMed]

10. Dolan, S.K.; O’Keefe, G.; Jones, G.W.; Doyle, S. Resistance is not futile: Gliotoxin biosynthesis, functionality and utility. Trends. Micobiol. 2015, 23, 419-428. [CrossRef] [PubMed]

11. Frisch, M.J.; Trucks, G.W.; Schlegel, H.B.; Scuseria, G.E.; Robb, M.; Cheeseman, J.R.; Scalmani, G.; Barone, V.; Mennucci, B.; Petersson, G.A.; et al. Gaussian 09, Revision D.01; Gaussian, Inc.: Wallingford, CT, USA, 2013.

12. Wu, P.; Xue, J.; Yao, L.; Xu, L.; Li, H.; Wei, X. Bisacremines E-G, three polycyclic dimeric acremines produced by Acremonium persicinum SC0105. Org. Lett. 2015, 17, 4922-4925. [CrossRef] [PubMed]

13. Bruhn, T.; Schaumlöffel, A.; Hemberger, Y.; Bringmann, G. SpecDis: Quantifying the comparison of calculated and experimental electronic circular dichroism spectra. Chirality 2013, 25, 243-249. [CrossRef] [PubMed]

14. Skehan, P.; Storeng, R.; Scudiero, D.; Monks, A.; McMahon, J.; Vistica, D.; Warren, J.T.; Bokesch, H.; Kenney, S.; Boyd, M.R. New colorimetric cytotoxicity assay for anticancer-drug screening. J. Natl. Cancer Inst. 1990, 82, 1107-1112. [CrossRef] [PubMed]

(C) 2019 by the authors. Licensee MDPI, Basel, Switzerland. This article is an open access article distributed under the terms and conditions of the Creative Commons Attribution (CC BY) license (http://creativecommons.org/licenses/by/4.0/). 